\title{
MAGNETO-STATIC MODELING OF THE MIXED PLASMA BETA SOLAR ATMOSPHERE BASED ON SUNRISE/IMaX DATA
}

\author{
T. Wiegelmann ${ }^{1}$, T. Neukirch ${ }^{2}$, D. H. Nickeler ${ }^{3}$, S. K. Solanki ${ }^{1,4}$, V. Martínez Pillet ${ }^{5}$, and J. M. Borrero ${ }^{6}$ \\ ${ }^{1}$ Max-Planck-Institut für Sonnensystemforschung, Justus-von-Liebig-Weg 3, D-37077 Göttingen, Germany; wiegelmann@mps.mpg.de \\ ${ }^{2}$ School of Mathematics and Statistics, University of St. Andrews, St. Andrews KY16 9SS, UK \\ 3 Astronomical Institute, AV CR, Fricova 298, 25165 Ondrejov, Czech Republic \\ ${ }^{4}$ School of Space Research, Kyung Hee University, Yongin, Gyeonggi, 446-701, Korea \\ ${ }^{5}$ National Solar Observatory, Sunspot, NM 88349, USA \\ ${ }^{6}$ Kiepenheuer-Institut für Sonnenphysik, Schöneckstrasse 6, D-79104 Freiburg, Germany \\ Received 2015 July 28; accepted 2015 October 23; published 2015 December 2
}

\begin{abstract}
Our aim is to model the three-dimensional magnetic field structure of the upper solar atmosphere, including regions of non-negligible plasma beta. We use high-resolution photospheric magnetic field measurements from SUNRISE/ $\mathrm{IMaX}$ as the boundary condition for a magneto-static magnetic field model. The high resolution of IMaX allows us to resolve the interface region between the photosphere and corona, but modeling this region is challenging for the following reasons. While the coronal magnetic field is thought to be force-free (the Lorentz force vanishes), this is not the case in the mixed plasma $\beta$ environment in the photosphere and lower chromosphere. In our model, pressure gradients and gravity forces are self-consistently taken into account and compensate for the non-vanishing Lorentz force. Above a certain height (about $2 \mathrm{Mm}$ ) the non-magnetic forces become very weak and consequently the magnetic field becomes almost force-free. Here, we apply a linear approach where the electric current density consists of a superposition of a field-line parallel current and a current perpendicular to the Sun's gravity field. We illustrate the prospects and limitations of this approach and give an outlook for an extension toward a nonlinear model.
\end{abstract}

Key words: methods: numerical - Sun: chromosphere - Sun: corona - Sun: magnetic fields - Sun: photosphere

\section{INTRODUCTION}

While the corona, at least above active regions, has a low plasma $\beta$ and is usually modeled by the assumption of a vanishing Lorentz force (see Wiegelmann \& Sakurai 2012, for an overview of solar force-free fields), this is not true in the lower solar atmosphere (see Wiegelmann et al. 2014, for a recent review on magnetic fields in the solar atmosphere). In the photosphere and lower chromosphere, low- and high- $\beta$ regions exist side by side and non-magnetic forces have to be taken into account to the lowest order with a magneto-static model where the Lorentz force is compensated for by the gradient of the plasma pressure and the gravity force.

The most accurate measurements of the solar magnetic field are available in the photosphere. In active regions, the full magnetic vector can be measured accurately, e.g., with $S D O /$ HMI, whereas in quiet Sun regions only the line of sight or vertical field is available with sufficient accuracy for a reliable extrapolation because in weak-field regions there is too much uncertainty in the transverse field components. (Noise in the Stokes vector translates into an uncertainty in the inferred values for the magnetic field; see Borrero \& Kobel 2011, 2012.) These photospheric measurements are extrapolated into the solar atmosphere under certain model assumptions, here using a magneto-static approach. The vertical resolution of the model scales with the horizontal resolution of the photospheric measurements, e.g., about $1400 \mathrm{~km}$ for $\mathrm{SOHO} / \mathrm{MDI}$-magnetograms and $350 \mathrm{~km}$ for $S D O / \mathrm{HMI}$. As the non-force-free layer containing the photosphere and lower chromosphere is rather thin (typically less than $2000 \mathrm{~km}$ ), one can hardly resolve magnetic structures here for models using $\mathrm{SOHO} / \mathrm{MDI}-$ or $S D O /$ HMI-magnetograms as the boundary condition. The high-resolution magnetograms from SUNRISE/IMaX with a pixel size of only $40 \mathrm{~km}$ now allow us to model this layer vertically with about 50 points.

A special class of magneto-static solutions which allow for separable solutions has been proposed by Low (1991). An advantage of this approach is that the resulting equations are linear (for nonlinear cases, see Neukirch 1997) and can be solved effectively by a Fourier transformation or a Green's function implementation (see Petrie \& Neukirch 2000). Separable and linear solutions have also been found in spherical (Bogdan \& Low 1986; Neukirch 1995; Al-Salti \& Neukirch 2010) as well as cylindrical coordinates (Neukirch 2009; AlSalti et al. 2010). The solutions found in spherical coordinates especially have been used for modeling the global magnetic field of the Sun (e.g., Bagenal \& Gibson 1991; Gibson \& Bagenal 1995; Gibson et al. 1996; Zhao et al. 2000; Ruan et al. 2008) and other stars (e.g., Lanza 2008, 2009).

Usually, these models require only the line of sight or vertical photospheric magnetic field as boundary condition, and the solutions contain free parameters and/or free functions. Nonlinear magneto-static solutions are more demanding numerically and observationally because they require photospheric vector magnetograms as input (see Wiegelmann \& Neukirch 2006; Wiegelmann et al. 2007, for a cartesian and spherical implementation, respectively). Within this work, we apply the linear magneto-static solutions proposed by Low (1991) to a high-resolution magnetogram observed with SUNRISE/IMaX. The outline of the paper is as follows. In Section 2, we briefly discuss the basic equations and model assumptions. Section 3 describes the employed photospheric magnetograms, which we use as boundary condition for our magneto-static model in Section 4. In Section 5, we finally discuss the prospects and limitations of this approach and 
provide an outlook for a generalization of the method toward a nonlinear numerical approach.

\section{BASIC EQUATIONS}

We use the magneto-hydro-static (MHS) equations

$$
\begin{gathered}
\boldsymbol{j} \times \boldsymbol{B}=\nabla P+\rho \nabla \Psi, \\
\nabla \times \boldsymbol{B}=\mu_{0} \boldsymbol{j}, \\
\nabla \cdot \boldsymbol{B}=0,
\end{gathered}
$$

where $\boldsymbol{B}$ is the magnetic field, $\boldsymbol{j}$ is the electric current density, $P$ is the plasma pressure, $\rho$ is the mass density, $\Psi$ is the gravitational potential, and $\mu_{0}$ is the permeability of free space. To find separable solutions for this set of equations, we apply the following ansatz for the electric current density (see Low 1991, for details):

$$
\nabla \times \boldsymbol{B}=\alpha_{0} \boldsymbol{B}+f(z) \nabla B_{z} \times \boldsymbol{e}_{z},
$$

where $\alpha_{0}$ is the force-free parameter and $f(z)$ is a free function which controls the non-magnetic forces. The first part, $\alpha_{0} \boldsymbol{B}$, corresponds to a field-line-parallel linear force-free current and the second term, $f(z) \nabla B_{z} \times \boldsymbol{e}_{z}$, defines a current perpendicular to the gravitational force (in the $z$-direction) or, in other words, parallel to the Sun's surface $(x, y)$. It is then possible to reduce the MHS equations to a single partial differential equation (see, e.g., Neukirch \& Rastätter 1999, for a particularly simple formulation) that can often be solved by the separation of variables. For convenience, here we use (as proposed in Low 1991)

$$
f(z)=a \exp (-\kappa z),
$$

with a free parameter $a$ which controls the non-magnetic forces in the photosphere. We note that $\kappa$ in Equation (5) controls the non-magnetic forces and should not be confused with the scale height of the plasma pressure. Obviously, for the choice of $a=0$, this approach reduces to linear force-free fields. Above a certain height in the solar atmosphere, one expects that the solution will become approximately force-free due to the low plasma $\beta$ in the solar corona. Consequently, $f(z)$ has to decrease with height and here we choose as the scale height the distance of the upper chromosphere above the solar surface, leading to $1 / \kappa=2 \mathrm{Mm}$. With $\kappa$ fixed, our MHS solution contains two free parameters, $\alpha$ and $a$.

As boundary conditions we use the measured vertical magnetic field $B_{z}(x, y, 0)$ in the photosphere. We solve the equations by means of a Fast-Fourier-Transform method similar to the linear force-free model developed by Alissandrakis (1981). A main difference from the linear force-free approach is that the resulting Schrödinger equation for $B_{z}$ in Fourier space contains a Bessel function instead of an exponential function.

One finds the following solution for pressure and density (see Low 1991, for the derivation):

$$
\begin{gathered}
P=P_{0}(z)-\frac{1}{2 \mu_{0}} f(z) B_{z}^{2}, \\
\rho=-\frac{1}{g} \frac{d P_{0}}{d z}+\frac{1}{\mu_{0} g}\left[\frac{d f}{d z} \frac{B_{z}^{2}}{2}+f(\boldsymbol{B} \cdot \nabla) B_{z}\right] .
\end{gathered}
$$

The first term in Equation (6) contains a 1D solution (in the $z$ direction) that is independent of the magnetic field and must obey $\nabla P=-\rho \nabla \Psi$. The second term is the disturbance of this $1 \mathrm{D}$ pressure profile by the magnetic field. Here, the pressure and density compensate for the non-vanishing Lorentz force. This disturbance is negative (if $a>0$ ) and obtains its highest absolute values in regions of the highest vertical magnetic field strength $B_{z}$. Because the total plasma pressure (sum of both terms) has to be positive, we obtain the following inequality for $P_{0}(z)$ :

$$
P_{0}(z)>a \cdot \exp (-k z) \cdot \frac{\operatorname{Max}\left(B_{z}\right)^{2}}{2 \mu_{0}}(z)
$$

where $\frac{\operatorname{Max}\left(B_{z}\right)^{2}}{2 \mu_{0}}(z)$ is the maximum at a given height $z$. As we will see later, this condition has severe consequences for an application to data with strong locally enhanced magnetic fields in the photosphere. To satisfy condition (8) in these regions, the background pressure $P_{0}$ has to be so high that the plasma $\beta$ in weak-field regions (and also on average) becomes unrealistically high, see Figure 3. Within this limitation, the choice of $P_{0}(z)$ has some freedom. Our choice is given in Section 4.1.

\section{DATA}

We apply our newly developed code to the photospheric magnetic field measurements taken with the balloon-borne SUNRISE solar observatory in 2009 June. For an overview of the SUNRISE mission and scientific highlights of the first SUNRISE flight see Solanki et al. (2010), Barthol et al. (2011), Berkefeld et al. (2011), and Gandorfer et al. (2011). For a description of the IMaX instrument, we refer to Martínez Pillet et al. (2011). The photospheric magnetic field was computed by inverting the IMaX measurements using the VFISV code as described in Borrero et al. (2011). The linear force-free extrapolation code, and the particular case of an $\alpha=0$ potential field has been applied to data from SUNRISE/IMaX before for a single magnetogram by Wiegelmann et al. (2010) and to analyze a time series by Wiegelmann et al. (2013). Chitta et al. (2014) carried out nonlinear force-free extrapolations from IMaX magnetograms and added vertical flows at low heights to simulate non-force-free effects in the photosphere and chromosphere. Here, we apply our newly developed linear MHS-code to a snapshot of the quiet Sun, also observed with SUNRISE/IMaX. We apply our code first to the full field of view of IMaX, as shown in Figure 1, and in a subsequent step we investigate a subfield (marked with a black rectangular in Figure 1) in more detail. The data set used here was observed in a period of $1.616 \mathrm{hr}$ starting at 00:00 UT on 2009 June 9 (image 220 from this series), see Martínez Pillet et al. (2011).

\section{RESULTS}

\subsection{Application to the Full IMaX-FOV}

In our first computation, we apply our model to the full phase-diversity reconstructed $\mathrm{IMaX}$ magnetogram of a quiet Sun region of $37 \times 37 \mathrm{Mm}$, which has been resolved by $936 \times 936$ pixels (pixel size on Sun $40 \mathrm{~km}$ ), see Figure 1. As our main interest lies in the mixed plasma $\beta$ regions of the photosphere and chromosphere, we extrapolate up to a height of $z=4 \mathrm{Mm}$ or 100 pixels. A few sample field lines for a magneto-static solution with $\alpha=3.0$ and $a=0.5$ are shown in in Figure 1(b). 

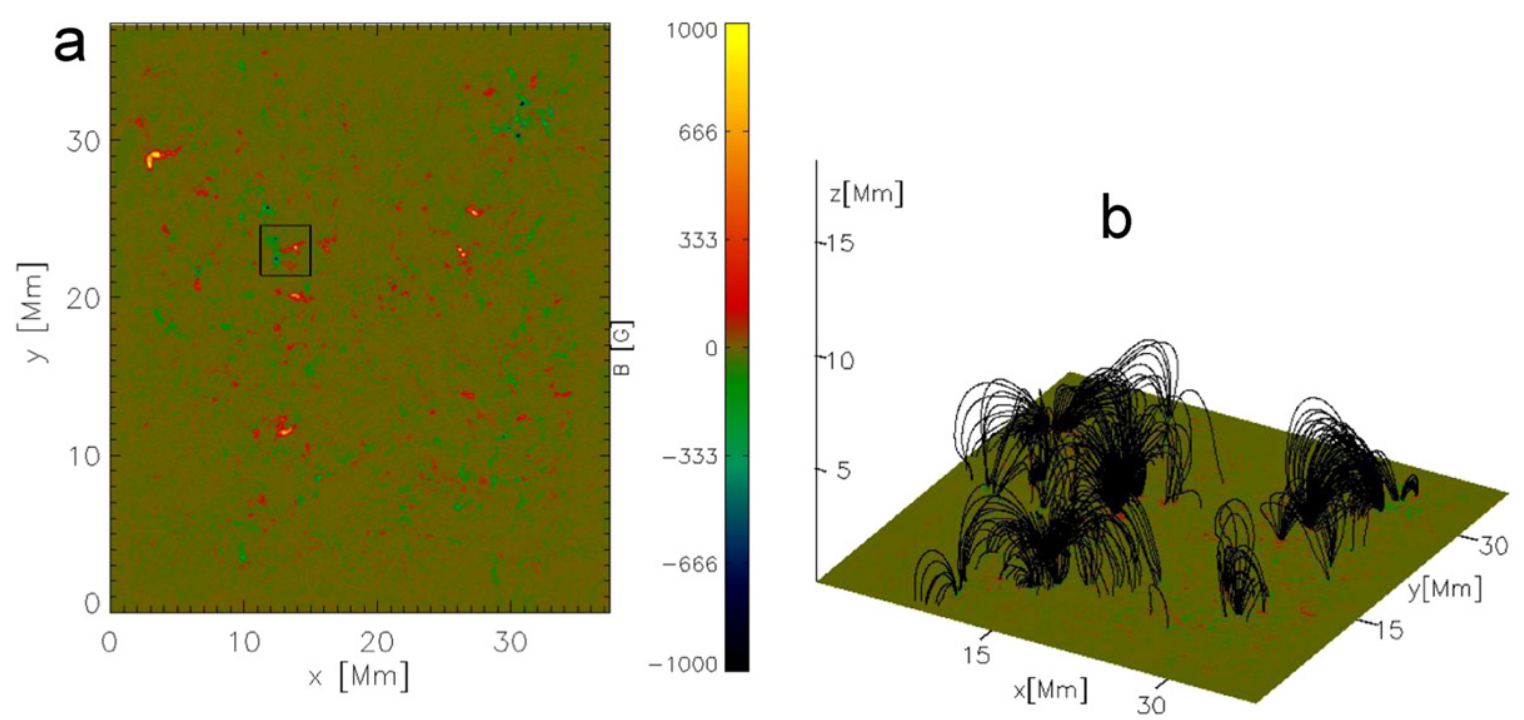

Figure 1. Panel (a): SUNRISE/IMaX magnetogram of a quiet Sun area. The black rectangular marks the region of interest. Panel (b): Sample field lines for a MHSmodel.
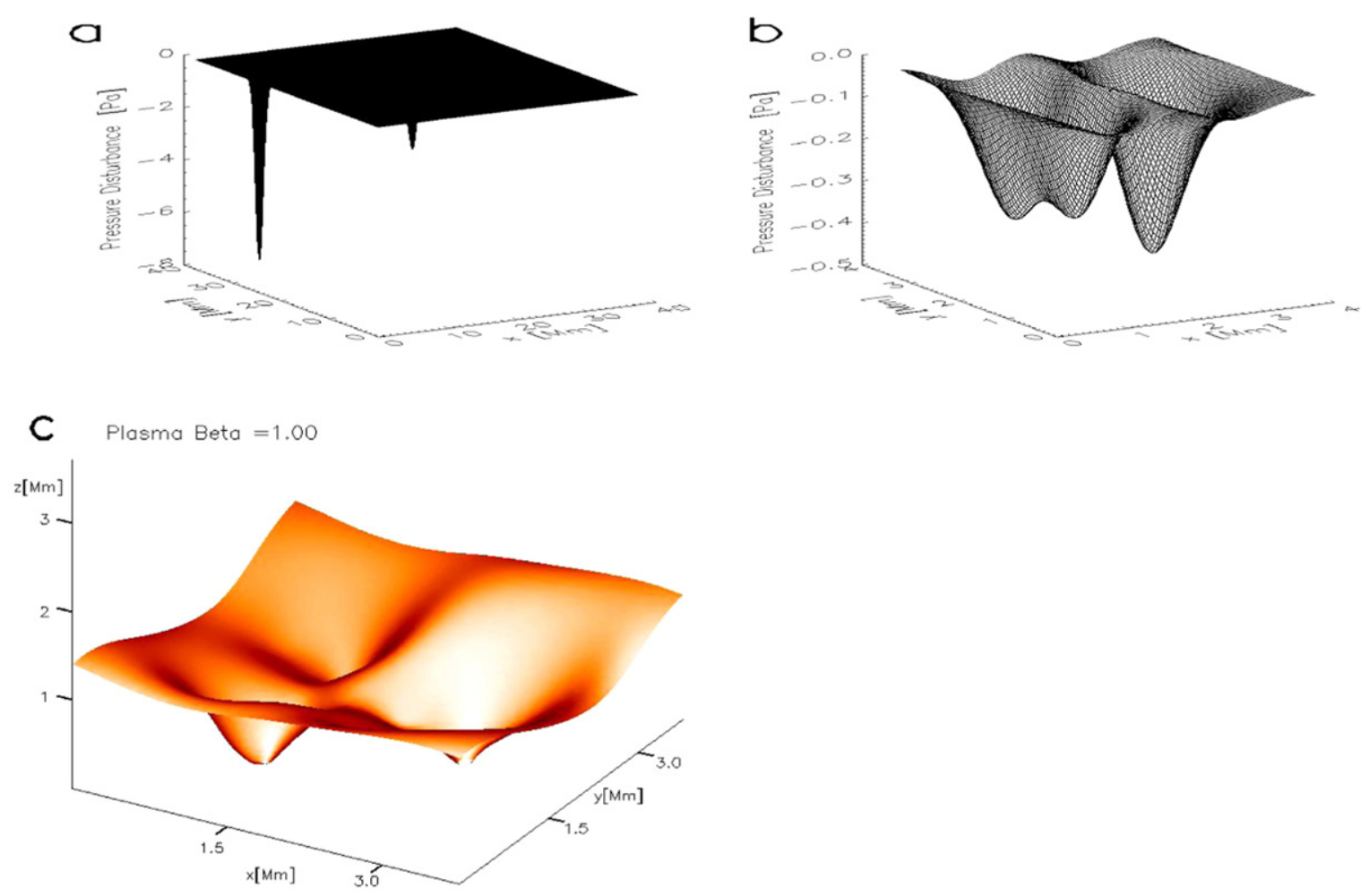

Figure 2. Plasma pressure disturbance $-\frac{1}{2 \mu_{0}} f(z) B_{z}^{2}$ at a height of $z=1 \mathrm{Mm}$ for the full IMaX and the small FOV in panels (a) and (b), respectively. Panel (c) shows an equi-contour surface for $\beta=1$ in the small FOV.

In Figure 2(a), we show the pressure disturbance in the chromosphere at a height of $z=1 \mathrm{Mm}$ as calculated with the second term $-\frac{1}{2 \mu_{0}} f(z) B_{z}^{2}$ on the right-hand side of Equation (6). This term obviously becomes largest above those regions with the highest photospheric field strength, as seen in the large negative peaks. Of course, the total pressure has to be positive and consequently a lower bound for the 1D background pressure $P_{0}$ is given by Equation (8). $P_{0}$ describes a 1D equilibrium between the gravity force and the vertical pressure gradient. One has to solve

$$
\frac{d P_{0}(z)}{d z}=-g \rho(z)
$$

for a constant gravity $g$. Assuming an equation of state of the form $P_{0}=\rho R T$, we get

$$
\frac{d P_{0}(z)}{d z}=-\frac{g P_{0}(z)}{R T}
$$




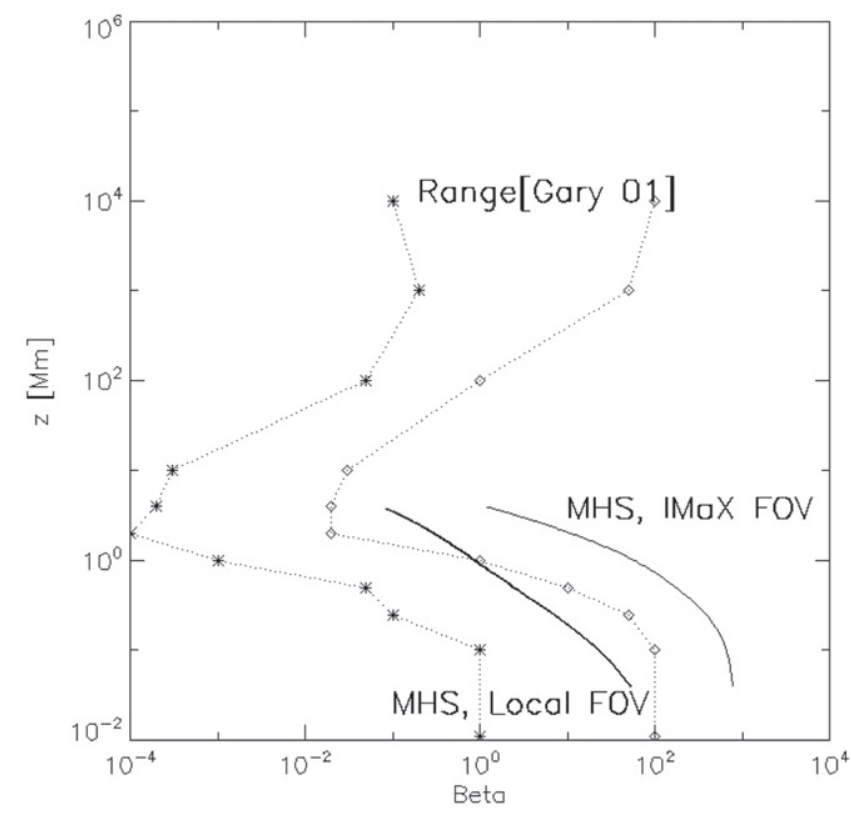

Figure 3. Plasma $\beta$ in the solar atmosphere. The dotted lines are taken from Gary (2001). The thin solid line shows the (horizontally averaged) plasma $\beta$ profile computed with our MHS model for the full IMaX FOV and the thick solid line represents the same for the selected small area.

which leads to the well-known atmospheric exponential decay ( $\propto \exp \left(-\frac{z}{2 H}\right)$, with $\left.H \approx 180 \mathrm{~km}\right)$ for a constant temperature of $T=T_{0}$, which is, however, not realistic for describing structures reaching from the photosphere through the chromosphere and into the corona. Equation (10) can be (numerically) integrated for any choice of a temperature profile $T(z)$, e.g., from 1D models of the solar atmosphere. Another alternative (because we already computed the 3D magnetic field from Equations (4) and (5)) is to prescribe the average plasma $\beta(z)$ as a function of z, e.g., from the literature (Gary 2001), leading to

$$
P_{0}(z)=\frac{\beta(z) B_{\mathrm{ave}}^{2}}{2 \mu_{0}},
$$

where $B_{\text {ave }}(z)$ is the horizontally averaged $B_{z}(x, y, z)$. The allowed ranges for $\beta(z)$ are bounded from below, however, by Equation (8). A choice which ensures a total positive pressure is obtained by using Equation (8) directly

$$
P_{0}(z)=P_{\epsilon}(z)+a \cdot \exp (-k z) \cdot \frac{\operatorname{Max}\left(B_{z}\right)^{2}}{2 \mu_{0}}(z),
$$

where $P_{\epsilon}(z)$ is the (prescribed) minimum value of the total pressure at a given height. For $P_{\epsilon}(z)=0$, the total plasma pressure becomes zero at the maximum of $B_{z}$ and remains positive elsewhere. Taking this into account, we can calculate the full average plasma $\beta$ (including the pressure disturbance) from Equation (6), as shown in Figure 3 by the rightmost curve labeled MHS, IMaX-FOV in Figure 3. The limitations from Equation (8) and a magnetogram with some high peak values in an otherwise weak-field region cause values of plasma $\beta$ which are too high and outside the range given by Gary (2001; dotted curves). We have to conclude that the linear MHS model cannot realistically be applied to the whole FOV of the SUNRISE magnetogram. The reason for this is that through Equation (12) the 1D background pressure, and thereby the maximum pressure in weak-field regions, is coupled with the highest values in the photospheric magnetogram, which is not very realistic.

\subsection{Application to a Small Part of the FOV}

Due to the difficulties involved with applying the linear MHS model to a full magnetogram, we restrict our analysis in the following to the smaller sub-region marked by the black rectangle in Figure 1(a). Figure 4 shows a few sample field lines for (a) a potential field model, (b) a linear force-free model with $\alpha=3$, and (c) a magneto-static solution $\alpha=3, a=0.5$. In the linear force-free case, the field lines become sheared compared with the potential field, and for some lines the connectivity changes. The influence of a nonvanishing Lorentz force (but using the same value of $\alpha$ as in the linear force-free case) has additional effects, which seem, however, to be smaller. The maximum heights of the loops are somewhat reduced and some additional field lines change their connectivity, e.g., in the MHS model no lines are connected with the positive (red) flux region close to the front boundary. Compared with the potential fields, the number of field lines connecting to this region was already reduced in the linearforce-free model.

The pressure disturbance in this smaller FOV is shown in the center panel of Figure 2. The absence of strong peaks in the photospheric field in this region leads to a much smoother distribution of the pressure disturbance. We use Equation (12) to compute the background pressure, and in Figure 3 the solid line marked MHS, local FOV shows the averaged plasma $\beta$ as a function of the height. At least in the photosphere and chromosphere, the plasma $\beta$ is within the limitation given by the dashed lines from the literature (Gary 2001). The true 3D plasma $\beta$ distribution, however, is not a function of $z$ only, but varies significantly in the horizontal direction. Figure 2(c) shows the equi-contours for $\beta=1.0$. As one can see, the $\beta=1.0$ surface is by no means plane-parallel, but is strongly corrugated. This behavior impacts methods for extrapolating force-free fields. Traditionally and for numerical simplicity, one extrapolates from a plane parallel surface (or the Sun's spherical surface) by assuming that the field above this lower boundary of the computational domain is force-free. In reality, however, the force-free domain is also bounded below by a corrugated surface. This is also true for planned measurements of the chromospheric magnetic field vector with Solar-C, and so magnetic field extrapolation techniques bounded by nonplane-parallel surfaces should be developed. In the non-forcefree region between the photosphere and the corrugated chromosphere, plasma pressure and gravity must be taken into account.

\section{DISCUSSION AND OUTLOOK}

The linear MHS approach used in this paper has two free parameters, the linear force-free parameter $\alpha$ and the force parameter $a$. Additionally, one also has to prescribe, in addition to the vertical magnetic field component at the lower boundary, the height in the solar atmosphere where the magnetic field becomes approximately force-free, here $1 / \kappa=2 \mathrm{Mm}$. Applying these solutions to large-scale areas has its limitations. These 

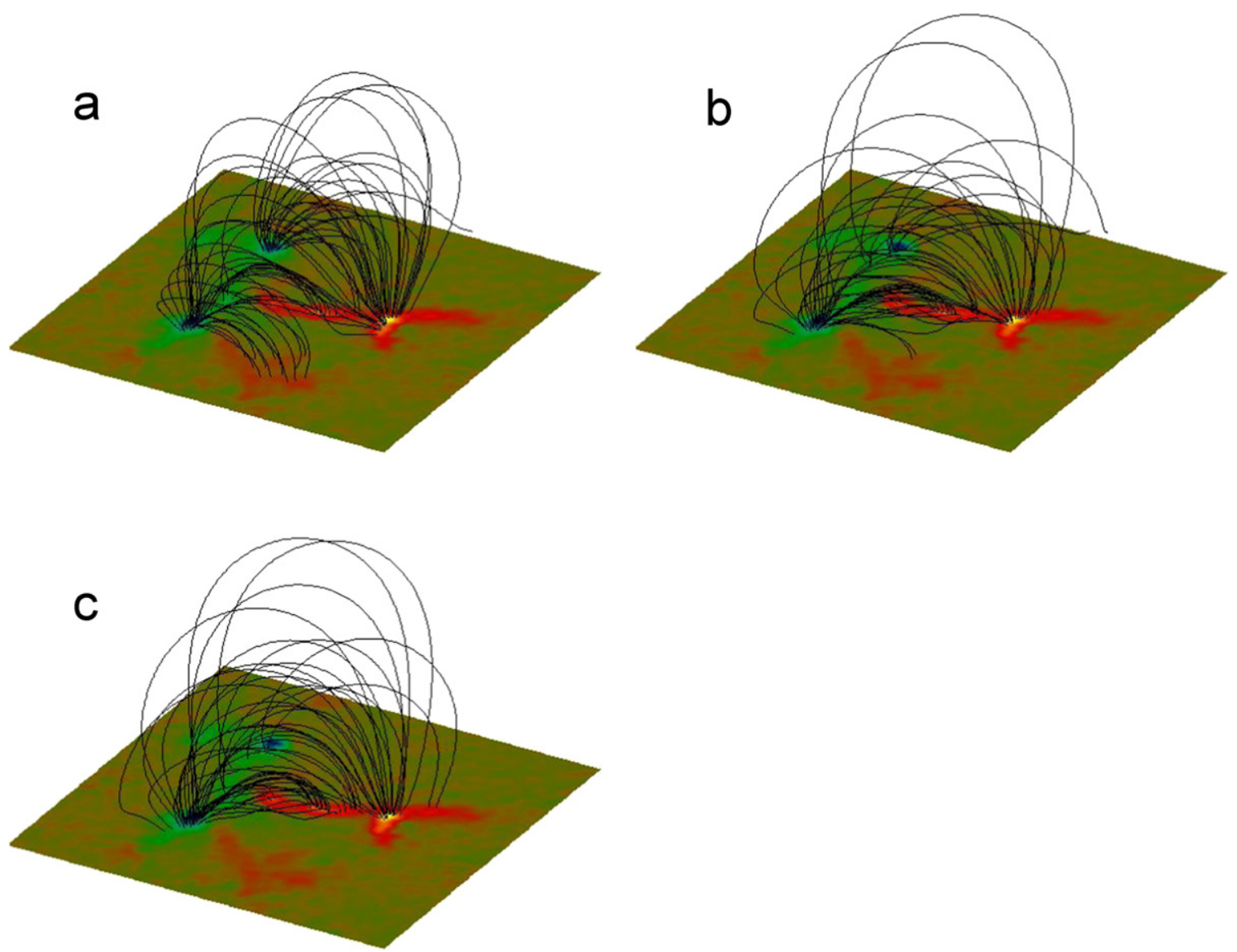

Figure 4. Small field of view (rectangular box in Figure 1(a)). (a) Potential field; (b) linear force-free field; (c) magneto-static field.

are, first of all, the well-known limitation of $\alpha$ being constant, which these solutions share with linear force-free configurations. Additionally, the pressure gradient (which compensates the Lorentz force) is coupled to the vertical magnetic field. As a consequence, the pressure disturbance, which is negative, becomes very large above strong fields in the photosphere. In order to maintain a positive total pressure, the background plasma pressure must be so strong that the average plasma $\beta$ becomes too high. This limitation of the method has to do with the fact that the two free parameters $\alpha$ and $a$ have to be the same in the entire computational domain. This limitation is similar to that for linear force-free fields, where one has only one free parameter $\alpha$ which has to be globally constant. While linear force-free fields cannot be used to model force-free configurations containing strong current concentrations in part of the domain (leading to localized high values of $\alpha$ ), a similar restriction occurs here for the linear magneto-static approach. Strong magnetic elements in an otherwise weak-field magnetogram cannot be modeled by this class of MHS solutions.

These limitations do not occur, however, for applications to regions with smaller fields of view because the assumption that $\alpha$ and $a$ are constant is naturally more reasonable the smaller the investigated domain is. How should one proceed to derive global magneto-static solutions? One possibility would be to compute the solutions discussed here only locally (with different values of $\alpha$ and $a$ in different regions) and to merge these configurations together. This will, of course, lead to solutions which are not entirely self-consistent and to inconsistencies at the boundaries between the different regions.
Another idea would be to use a numerical scheme, e.g., an optimization approach as suggested by Wiegelmann \& Neukirch (2006) and Wiegelmann et al. (2007), to relax these merged solutions toward a self-consistent (nonlinear) MHSequilibrium. Wiegelmann \& Neukirch (2006) and Wiegelmann et al. (2007) developed nonlinear magneto-static codes in Cartesian and spherical geometry, respectively. For the smallscale features measured with SUNRISE, one would naturally apply the cartesian version. These codes require photospheric vector magnetograms as input, which are not available with the required quality for the investigated quiet Sun region because of the poor signal-to-noise ratio (for horizontal fields) in weakfield regions. Nonlinear approaches (both force-free and magneto-static) are well suited to dealing with local strong enhancements (e.g., current concentrations and strong flux elements). It is a weakness of any linear approach that they cannot deal with strong localized enhancements of relevant quantity.

To be able to carry out nonlinear magneto-static (or nonlinear force-free) extrapolations, measurements of the horizontal photospheric magnetic field would be helpful. During the re-flight of SUNRISE in 2013, high-resolution vector magnetograms of active region(s) were measured with IMaX. We plan to use these measurements for self-consistent nonlinear magneto-static modeling in our future work.

The German contribution to SUNRISE is funded by the Bundesministerium für Wirtschaft und Technologie through Deutsches Zentrum für Luft- und Raumfahrt e.V. (DLR), grant 
No. 50 OU 0401, and by the Innovationsfonds of the President of the Max Planck Society (MPG). The Spanish contribution has been funded by the Spanish MICINN under projects ESP2006-13030-C06 and AYA2009-14105-C06 (including European FEDER funds). The HAO contribution was partly funded through NASA grant number NNX08AH38G. T.N. acknowledges support by the U.K.'s Science and Technology Facilities Council and would like to thank the MPS for its hospitality during a visit in 2014 December. D.H.N. acknowledges financial support from GA ČR under grant number 13$24782 \mathrm{~S}$. The Astronomical Institute Ondrejov is supported by the project RVO:67985815. This work was partly supported by the BK21 plus program through the National Research Foundation (NRF) funded by the Ministry of Education of Korea.

\section{REFERENCES}

Alissandrakis, C. E. 1981, A\&A, 100, 197

Al-Salti, N., \& Neukirch, T. 2010, A\&A, 520, A75

Al-Salti, N., Neukirch, T., \& Ryan, R. 2010, A\&A, 514, A38

Bagenal, F., \& Gibson, S. 1991, JGR, 96, 17663

Barthol, P., Gandorfer, A., Solanki, S. K., et al. 2011, SoPh, 268, 1

Berkefeld, T., Schmidt, W., Soltau, D., et al. 2011, SoPh, 268, 103

Bogdan, T. J., \& Low, B. C. 1986, ApJ, 306, 271
Borrero, J. M., \& Kobel, P. 2011, A\&A, 527, A29

Borrero, J. M., \& Kobel, P. 2012, A\&A, 547, A89

Borrero, J. M., Tomczyk, S., Kubo, M., et al. 2011, SoPh, 273, 267

Chitta, L. P., Kariyappa, R., van Ballegooijen, A. A., DeLuca, E. E., \& Solanki, S. K. 2014, ApJ, 793, 112

Gandorfer, A., Grauf, B., Barthol, P., et al. 2011, SoPh, 268, 35

Gary, G. A. 2001, SoPh, 203, 71

Gibson, S. E., \& Bagenal, F. 1995, JGR, 100, 19865

Gibson, S. E., Bagenal, F., \& Low, B. C. 1996, JGR, 101, 4813

Lanza, A. F. 2008, A\&A, 487, 1163

Lanza, A. F. 2009, A\&A, 505, 339

Low, B. C. 1991, ApJ, 370, 427

Martínez Pillet, V., Del Toro Iniesta, J. C., Álvarez-Herrero, A., et al. 2011, SoPh, 268, 57

Neukirch, T. 1995, A\&A, 301, 628

Neukirch, T. 1997, A\&A, 325, 847

Neukirch, T. 2009, GApFD, 103, 535

Neukirch, T., \& Rastätter, L. 1999, A\&A, 348, 1000

Petrie, G. J. D., \& Neukirch, T. 2000, A\&A, 356, 735

Ruan, P., Wiegelmann, T., Inhester, B., et al. 2008, A\&A, 481, 827

Solanki, S. K., Barthol, P., Danilovic, S., et al. 2010, ApJL, 723, L127

Wiegelmann, T., \& Neukirch, T. 2006, A\&A, 457, 1053

Wiegelmann, T., Neukirch, T., Ruan, P., \& Inhester, B. 2007, A\&A, 475, 701

Wiegelmann, T., \& Sakurai, T. 2012, LRSP, 9, 5

Wiegelmann, T., Solanki, S. K., Borrero, J. M., et al. 2010, ApJL, 723, L185

Wiegelmann, T., Solanki, S. K., Borrero, J. M., et al. 2013, SoPh, 283, 253

Wiegelmann, T., Thalmann, J. K., \& Solanki, S. K. 2014, A\&ARv, 22, 78

Zhao, X. P., Hoeksema, J. T., \& Scherrer, P. H. 2000, ApJ, 538, 932 